\title{
HIGHER EDUCATION RESEARCH PROJECT: LA VISIÓN DE SEIS PAÍSES DESARROLLADOS SOBRE LA EDUCACIÓN SUPERIOR NECESARIA PARA EL NUEVO MILENIO
}

\author{
Flora Eugenia Salas Madriz \\ Docente de la Escuela de Administración Educativa \\ de la Universidad de Costa Rica
}

Recibido 30-VIII-2004 • Aceptado 7-IX-2004

\begin{abstract}
Resumen: En este artículo se ofrece una sintesis de los temas y conclusiones de la conferencia Higher Education Reform for Quality Higher Education Management in the 21th. Century. Economical, Technological, social and political Forces Affecting Higher Education, que se realizó en Hiroshima, Japón, en 1999, debido a que en ella se discutió la situación de la educación superior de China, Alemania, Japón, Singapur, Suiza y los Estados Unidos, y sus principales problemas, las áreas estratégicas, las prioridades y las alternativas de acción necesarias para ajustar sus sistemas universitarios a los requerimientos científicos y tecnológicos, al mercado de trabajo y a la cultura propios de la Sociedad del Conocimiento.
\end{abstract}

Palabras clave: Educación superior, globalización y universidad, sociedad del conocimiento y educación superior, cambio en educación superior, administración universitaria.

Abstract: This article provides a synthesis of the topics and conclusions from the conference Higher Education Reform for Quality Higher Education Management in the 21st. Century. Economical, Technological, Social and Political Forces Affecting Higher Education. This conference took place in Hiroshima, Japan, in 1999. The current situation of higher education in China, Germany, Japan, Singapore, Switzerland and the United States was discussed in this conference which also dealt with issues such as strategic areas, priorities and alternative actions necessary to adjust these university syste$m s$ to the scientific and technological requirements of the job market in the society of knowledge.

Key words: Higher education, globalization and university, society of knowledge and higher education, changes in higher education, university administration.

\section{Introducción}

La década de los 90 fue decisiva para la educación superior, ya que en 1998, como conclusión de encuentros preliminares en todos los continentes, se realizó en París, auspiciada por la UNESCO, la conferencia mundial sobre La Educación superior en el siglo XXI. En este foro se discutió la situación de la educación superior y se trazó la visión global del papel que deben jugar las universidades en el contexto de la globalización y la revolución de las tecnologías de la información y la comunicación (TIC); ambos, acontecimientos que alteraron en forma radical el sistema político, económico, social, científico y cultural del mundo conocido hasta comienzos de la década de los 90. Con base en los resultados de esta conferencia, se iniciaron procesos de cambio e innovación de la educación superior en las distintas regiones y subregiones del mundo con el propósito de dar respuesta a las nuevas demandas socioeconómicas y educativas del postcapitalismo.

En este nuevo contexto, se considera oportuno exponer la visión de la educación superior en seis de los países más ricos y desarrollados del mundo que se caracterizan, además, por su elevada producción de conocimiento científico y tecnología: 
China, Alemania, Japón, Singapur, Suiza y los Estados Unidos. Estas naciones se unieron en la década de los 90, para poner en marcha un ambicioso proyecto de cooperación para el desarrollo de la educación a partir de la investigación, denominado Six Nation Project, con el propósito de que sus sistemas educativos respondan a los acelerados cambios y nuevos requerimientos de la sociedad del siglo XXI.

A continuación, se hace una breve reseña del origen y principales acontecimientos del proyecto. Posteriormente, se ofrece una síntesis de los temas y conclusiones de la conferencia Higher Education Reform for Quality Higher Education Management in the 21th. Century. Economical, Technological, social and political Forces Affecting Higher Education, que se realizó en Hiroshima, Japón, en 1999, debido a que en ella se discutió la situación de la educación superior en estos países, sus principales problemas, las áreas estratégicas, las prioridades y las alternativas de acción necesarias para ajustar sus sistemas universitarios a los requerimientos científicos y tecnológicos, al mercado de trabajo y a la cultura propios de la Sociedad del Conocimiento.

Esperamos que los resultados de este esfuerzo de cooperación para el mejoramiento de la educación superior de seis países desarrollados, sirvan para dar una perspectiva general de los procesos de reforma, innovación y cambio que se están llevando a cabo en educación superior países desarrollados, así como para reflexionar sobre las tareas aún pendientes y las acciones necesarias para lograr que las universidades contemporáneas se adapten a los requerimientos educativos en la Era de la Globalización y la Información.

\section{El Proyecto Seis Naciones}

En diciembre de 1993, en Pennsylvania, Estados Unidos, se llevó a cabo una reunión de representantes de los gobiernos y del sector educativo de China, Alemania, Japón, Singapur, Suiza y los Estados Unidos, con el propósito de discutir la posibilidad de desarrollar un programa de cooperación para el desarrollo de la investigación educativa. El proyecto se denominó SixNation Education Research Program. Como resultado de esa primera reunión se creó un comité especial para dar forma al proyecto, el cual convocó a una segunda conferencia, también en Pennsylvania, en junio de 1995, para definir líneas de acción, así como eventuales compromisos de cada país para el logro de las metas previstas en el primer encuentro. En esta segunda reunión cada nación definió las áreas de investigación en las que tenía interés a fin de determinar cuáles de ellas eran compartidas por los seis países. Las áreas en cuestión fueron las siguientes:

- China: Programas, políticas y proyectos educativos.

- Alemania: Educación y crecimiento económico.

- Japón: Educación Superior.

- $\quad$ Singapur: Enseñanza del lenguaje y capacidad lecto-escritora.

- Suiza: Educación vocacional.

- Estados Unidos: Enseñanza de las ciencias y las matemáticas.

Como se puede observar, si bien el proyecto originalmente tuvo como propósito el mejoramiento de la educación en general, se partió del supuesto de que esta tarea debía ser asumida por las universidades, en el entendido que, gracias a su misión y características, estas instituciones tienen tanto la responsabilidad como la posibilidad de favorecer la producción del conocimiento científico y la búsqueda permanente del desarrollo y progreso de la sociedad. Después de estas dos reuniones, hubo otros encuentros del comité organizador para definir líneas de acción en Singapur (1996), Suiza (1997) y China (1999).

En el campo de la educación superior se creó un proyecto específico, denominado Higher Education Research Project, y 
se realizó un seminario internacional en la Universidad de Hiroshima en 1997. El tema de este primer seminario fue Academic Reforms in the World: Situation and Perspective in the Massification Stage of Higher Education, que sirvió de base para la conferencia de 1999. Esta última conferencia también se llevó a cabo en la Universidad de Hiroshima, Japón, y su tema fue Higher Education Reform for Quality Higher Education Management in the 21th. Century. Economical, Technological, social and political Forces Affecting Higher Education. A ella asistieron 188 representantes de los países miembros del proyecto, 22 invitados especiales entre los que se contó con la participación de investigadores de reconocimiento mundial en el campo de la educación superior, rectores de universidades, ejecutivos de empresas, y representantes gubernamentales. Además, participaron 600 personas de distintos ámbitos y sectores de Japón. Los temas y conclusiones de la misma, se presentan a continuación.

\section{Temas y conclusiones de la conferencia Higher Education Reform for Quality Higher Education Management in the 21th. Century. Economical, Technological, social and political Forces Affecting Higher Education}

Con base en los resultados del seminario de 1997 (Academic Reforms in the Word: Situation and Perspective in the Massification Stage of Higher Education), se determinó que los temas a discutir en la conferencia Higher Education Reform for Quality Higher Education Management in the 21th. Century. Economical, Technological, social and political Forces Affecting Higher Education, serían los siguientes ${ }^{1}$ :
- Marco de referencia de las políticas en Educación Superior.

- Gestión Estratégica para las universidades.

- Educación superior y sociedad.

- El papel de las universidades en el crecimiento económico; Educación superior y tecnología: ¿cuándo cambiará realmente la tecnología cómo se enseña y cómo aprenden los estudiantes en la universidad?

El Prof. Akira Arimoto de la Universidad de Hiroshima, abrió la conferencia con una ponencia en la que hizo un contexto general de la educación superior hasta 1999. Como punto de partida señaló que la administración universitaria desempeña un papel fundamental en el desarrollo de la educación superior para el siglo XXI, ya que para hacer los cambios necesarios para adecuar el quehacer de la universidad al nuevo contexto mundial, es impostergable una reforma académica profunda que implica una reorganización administrativa y gerencial substanciales. Subrayó, además, que para agregar complejidad al proceso, esta reorganización se debe hacer en medio de una situación crítica del financiamiento de las instituciones de educación superior, en un momento de rendición de cuentas y evaluación de las universidades, y frente a una creciente competencia por recursos y población estudiantil, debido al incremento acelerado de la oferta académica privada. Asimismo, señaló que a lo anterior se debe sumar el imperativo del desarrollo tecnológico en las universidades en el nivel administrativo y en la enseñanza.

Para comprender el presente es necesario tener en cuenta cuáles han sido las últimas fases de desarrollo de las universidades en el nivel mundial. De hecho, a nuestro parecer, la década de los 80 es un punto de inflexión en la historia reciente de esta institución debido al fenómeno de la masificación, consecuencia del incremento en la demanda por educación superior que se dio en ese período y que tuvo un 
comportamiento similar en todo el mundo. En esta década, el sector empleador, en general, elevó los requisitos de contratación de personal a los niveles técnicos y profesionales, razón por la cual tanto jóvenes como adultos se vieron en la necesidad de recibir formación universitaria y técnica. A su vez, los gobiernos tuvieron que responder a este incremento acelerado de la demanda por educación superior creando nuevas universidades y abriendo las posibilidades legales para la oferta académica de instituciones de educación superior privadas, ya que para los estados resultó imposible atender esta demanda con recursos e infraestructura públicos (Jofré, 1994).

Como resultado de la masificación de la educación superior, nuevos problemas emergieron en el escenario universitario. La reducción del tiempo de la formación profesional, el poco o ningún control estatal sobre el quehacer de estas instituciones, tanto estatales como privadas, y la creciente competencia entre universidades por recursos públicos y por estudiantes, inevitablemente trajo consigo una pérdida significativa de la calidad de la formación universitaria (Jofré, 1994; Tünnermann, 1997, 1999). Como se puede observar, a la crisis de la masificación, secuela de la fuerte presión social por más instituciones de educación superior, siguió la crisis de calidad; es decir, en un plazo de diez años, las naciones enfrentaron el crecimiento de una compleja trama de instituciones, títulos y carreras, tanto públicas como privadas, que no siempre cumplían con los requisitos mínimos para garantizar la formación de profesionales capaces de atender las tareas $\mathrm{y}$ funciones para los que posteriormente eran contratados.

Como consecuencia de la crisis de calidad de la educación superior, se implementaron medidas de control y evaluación universitaria en todo el mundo. En la década de los 90, se pusieron en práctica mecanismos de autoevaluación y autorregulación de las universidades, con el objetivo de “acreditar" las carreras y las instituciones.
Se crearon sistemas para auditorar las universidades públicas y privadas, así como mecanismos para controlar la creciente venta de servicios en las instituciones de educación superior públicas, que surgió como alternativa para resolver, al menos en parte, la reducción del financiamiento estatal. A este período también se le llama "posmasificación".

Con base en este marco de referencia, las universidades presentan características específicas, dependiendo de si se encuentran en la fase previa a la masificación, en la fase de masificación o en la fase de la posmasificación. A la fase previa a la masificación se le denomina "academia tipo élite", ya que en esta fase sólo acceden a la educación superior las clases económica y políticamente privilegiadas que, a su vez, tienen acceso a una buena educación primaria y secundaria. En la fase de la masificación, sectores medios y pobres tienen acceso a la educación secundaria, razón por la cual ingresa a la universidad una población más diversa en términos socioeconómicos y educativos. En la fase de la posmasificación se enfrenta la crisis de calidad propia de la diversidad, del crecimiento poco ordenado y desorganizado de las universidades, y del poco control sobre las instituciones de educación superior, tanto públicas como privadas. Dentro de esta clasificación, los países participantes se catalogaron de la siguiente forma:

- Academia tipo élite: China.

- Fase de masificación: Alemania, Suiza y Singapur.

- Fase de posmasificación: Estados Unidos y Japón.

La fase de masificación tiene distintos momentos de desarrollo que, según Teichler ${ }^{2}$, se pueden diferenciar a partir de la mayor o menor presencia de los siguientes factores:

1. El mejoramiento y extensión del desarrollo del cuerpo docente. 
2. Introducción de cursos modulares y sistemas de créditos.

3. Nuevas mezclas entre educación general y especializada.

4. Estudio orientado a la práctica.

5. Aumento de opciones académicas.

6. Énfasis en el desarrollo personal, flexibilidad y desarrollo de habilidades y destrezas sociales.

7. Mejoramiento y valoración del éxito académico. (Report of the Six-Nation Higher Education Project, 2000, p. 5).

Pese a los esfuerzos por resolver los problemas asociados a la masificación, las universidades que enfrentan esta fase tienen dificultades para garantizar la calidad de la formación profesional que ofrecen; por esta razón, ingresan en un nuevo proceso de reforma académica que es la fase a la que se denomina "posmasificación". Esta fase se caracteriza por la implementación de procesos de reforma y cambio académico, orientados a satisfacer la presión social y empresarial por formación profesional de calidad, así como las nuevas demandas educativas de estudiantes no tradicionales; es decir, de adultos graduados, ubicados laboralmente, que requieren procesos de capacitación y reciclaje profesional para elevar su competitividad en el sector laboral. A este proceso se le denomina "educación continua o a lo largo de la vida" que viene del término inglés long-life learning.

Los estudiantes no tradicionales son los estudiantes de tiempo parcial, los que pertenecen a minorías, mujeres, emigrantes, y estudiantes adultos. Para que las universidades puedan atender las necesidades educativas de esta población son indispensables el mejoramiento de la educación general, cambios curriculares, la garantía de la calidad de la enseñanza, y el desarrollo del personal docente y administrativo. Debido a las necesidades educativas de esta nueva población, que se suman a las de los estudiantes tradicionales -jóvenes entre los 18 y los 24 años, que estudian a tiempo completo en sistemas presenciales-, la universidad enfrenta los retos de ofrecer educación continua, superar los esquemas de formación tradicionales y acercarse a la comunidad y a los sectores productivos.

Además, en este escenario, es inevitable discutir el uso efectivo de la tecnología en una época donde se incrementa drásticamente el impacto de las TIC en todos los aspectos de la vida; por esta razón, en la fase de la posmasificación es necesario que las universidades se planteen de qué manera pueden introducir estas herramientas para mejorar la enseñanza, el aprendizaje e innovar el currículum, con el propósito de que la academia realmente contribuya a maximizar las ventajas del nuevo ambiente tecnológico que caracteriza la sociedad actual, y que probablemente se mantenga e, incluso, crezca y se complejice en el futuro.

De igual manera que es preciso conocer las fases de la educación superior en los últimos veinte años para poder tener una perspectiva clara de su rumbo en el futuro próximo, es necesario conocer el contexto social, económico, político, científico y cultural emergentes. Al respecto, el Prof. Kenneth P. Mortimer, de la Universidad de Hawai, en su ponencia titulada Governance in the 21th. Century University, destacó los siguientes cinco aspectos, pues considera que son fundamentales para comprender el escenario global en el que están inmersas hoy las universidades:

- La creciente importancia de las fuerzas del mercado.

- La globalización de la ciencia y la tecnología.

- La creciente importancia de la tecnología.

- $\quad$ El creciente énfasis en los resultados educativos.

- La integración de la universidad con la sociedad.

Las nuevas demandas del mundo del trabajo y las características de la producción y distribución de bienes y servicios de la 
economía de la Sociedad del Conocimiento, exigen una continua intervención en los procesos productivos de los centros que generan conocimiento e investigación para resolver los problemas relacionados con una sociedad y una economía altamente complejas. Por esta razón, el mercado está cobrando importancia en el quehacer de las universidades, ya que no sólo se están creando alianzas entre centros de investigación universitarios y empresas, sino que también se está configurando un nuevo "capitalismo académico", resultado de la venta de servicios altamente especializados, relacionados, como mencionamos anteriormente, con la investigación. Esta situación está modificando en forma radical las relaciones entre la universidad y el sector productivo y, también está acelerando cambios administrativos y la legislación interna de las universidades. Para algunas personas esta es una amenaza para la institución, porque determina y condiciona por medio de intereses económicos la investigación y el quehacer universitario, dejando de lado el carácter autónomo y crítico que ha caracterizado desde sus inicios la vida académica. Para otras, esta es una oportunidad sin precedentes para que las universidades se liberen de las limitaciones presupuestarias que las han restringido en las últimas décadas y una excelente ocasión para vincular la academia con el sector productivo.

Otro aspecto fundamental de la época actual es la creciente importancia de la tecnología y, en particular, de las tecnologías de la información y la comunicación, ya que gracias a estas últimas se eliminaron finalmente las barreras espacio-temporales para enseñar y aprender. Estas tecnologías son una oportunidad para dar respuesta a las necesidades educativas de la población estudiantil no tradicional -que es uno de los grandes retos para la universidad de nuestro tiempo-, y para mejorar la calidad de la enseñanza y el aprendizaje de los estudiantes tradicionales.

Para Mortimer, también resulta fundamental hacer un cambio radical en la manera como se evalúan los resultados educativos, pues considera que es condición de la transformación de la universidad superar la perspectiva heredada de la "academia elitista" que hace énfasis en los resultados, cuyas características son:

- Excesiva validación de la calidad de la institución por la reputación (se apela a una historia de reconocimiento de excelencia).

- Una alta selectividad en el proceso de admisión.

- Una mayoría de sus estudiantes son de tiempo completo.

- La evaluación de la calidad se centra en los resultados (rendimientos), y no considera el aprovechamiento real de la formación de los graduados en el nivel cualitativo y real. (Report of the Six-Nation Higher Education Project, 2000, p. 12).

Dar cuenta del aprovechamiento de la formación es fundamental para dar el salto cualitativo que se requiere en la enseñanza y el aprendizaje para que las personas que viven procesos de educación superior en nuestro tiempo desarrollen las habilidades, destrezas y actitudes necesarias para un adecuado desempeño individual y laboral en la Sociedad del Conocimiento. El modelo tradicional de enseñanza y aprendizaje debe ser superado.

Finalmente, señala Mortimer que participar a la sociedad civil de los procesos y decisiones educativas es una tarea ineludible de nuestro tiempo, ya que es necesario mejorar la experiencia de los estudiantes y en ello consiste, precisamente, mucho de la calidad de la educación superior que deseamos para el futuro. Sólo una formación integral es capaz de desarrollar la conciencia crítica y la inteligencia responsable que se requieren para contribuir al mejoramiento de la sociedad y al progreso de la cultura. Como se puede observar, para Mortimer el mercado, la globalización, las nuevas demandas de la sociedad civil y la tecnología 
son importantes y poderosas fuerzas para el cambio en educación superior.

En la misma línea de Mortimer, Ruiqing Du, de la Universidad de Lenguas Extranjeras de Xi'an, China, y Elizabeth A. Zinder de la Universidad de Kentucky, plantean en sus ponencias tituladas respectivamente, A system in Transition-Higher Education Policy y Reform for Quality Education in the 21th. Century: Policy and Future Plans from the Unites States Perspective, que nunca como en la década de los 90 la educación superior adquirió tanta relevancia en los niveles gubernamentales y públicos, ya que en la Sociedad del conocimiento se deben promover la investigación, el desarrollo de la ciencia y la tecnología y, en particular, aprovechar las tecnologías de la información y la comunicación para mejorar la academia en los niveles administrativo y académico.

Para Zinder, estamos en una fase de tensión entre la academia tradicional y las nuevas demandas sociales, y es necesario reconocer que las universidades de hoy no están organizadas para sacar ventajas rápidas y adecuadas de las disciplinas emergentes ni de las nuevas tecnologías. En una sociedad donde la brecha entre las personas con y sin educación superior se hace cada vez más grande, mientras las oportunidades por educación superior crecen, la universidad será juzgada por el impacto que tenga en la vida de las personas; por esta razón, para Zinder es necesario orientar las metas de las universidades a los clientes y a la sociedad. Al respecto, hizo referencia a la necesidad de un currículum centrado en el estudiante y a los resultados finales de la Conferencia Mundial sobre Educación Superior de la UNESCO (París, 1998), donde se concluyó que las nuevas tecnologías son un aspecto central en la búsqueda del incremento de la calidad, la pertinencia y la equidad en educación superior.

Makoto Nagao, de la Universidad de Kyoto, en su ponencia Present and Future of Higher Education in Japan, y Yoshuo Harada, de la Universidad de Hiroshima, en su ponencia Establishment of Efficient Management in a Institution of Higher Education, dirigieron su atención a los problemas de la universidad en el futuro cercano. Para Nagao, los más importantes son los siguientes:

- La internacionalización de las actividades de las universidades.

- $\quad$ El papel de las universidades en el desarrollo de la ciencia y la tecnología. El liderazgo e iniciativas de las universidades para reestablecer la "humanización" en una sociedad regida por la tecnología.

Nagao focalizó su intervención en las dimensiones ética y política de las universidades en una época donde los valores económicos aparecen como el "único valor". En este mismo sentido, Harada señala que la educación, en general, hoy enfrenta problemas profundos que no son exclusivos de un país o una región sino que son globales, como:

- $\quad$ El matonismo infantil y adolescente.

- El vandalismo.

- El desinterés infantil y adolescente por el estudio y el desconocimiento de la autoridad de los maestros.

- La pérdida creciente de autoridad de los maestros en clase.

Harada considera que la crisis de la educación primaria y secundaria, que se refleja en los factores mencionados, incide de manera negativa en la educación superior, ya que muchos de los estudiantes jóvenes que ingresan a la universidad en la actualidad aún no han desarrollado las habilidades, conocimientos y destrezas necesarios para continuar procesos de formación de orden superior, y tampoco tienen una actitud adecuada para asumir el reto personal de llevar a cabo actividades y tareas propias de un proceso educativo donde la responsabilidad por el propio aprendizaje se incrementa sustancialmente. Este tipo de población estudiantil, que 
crece año a año, incrementa los costos de la educación superior, impacta negativamente el desarrollo del currículum, eleva las tasas de repitencia y permanencia, y pone en riesgo el patrimonio material y la cultura de la institución.

Sirve de ejemplo a lo señalado por Nagao y Harada, la situación que se vive en muchas escuelas e instituciones secundarias de los Estados Unidos y otros países de la región -incluida Costa Rica-, donde no sólo hay antecedentes de matanzas perpetradas por estudiantes en escuelas y secundarias, sino también donde se ha vuelto una práctica común que los alumnos sean requisados para poder ingresar al centro educativo, ya que algunos portan drogas, material pornográfico, material ofensivo o peligroso, e, incluso, armas de fuego, explosivos y objetos punzo-cortantes.

Si bien Nagao y Harada tocan algunos de los problemas éticos y políticos de la educación de nuestro tiempo, no hacen referencia al problema de la corrupción que es endémico en las naciones latinoamericanas y Costa Rica, lamentablemente, tampoco es la excepción. Nos parece importante incluir en esta discusión ética que muchos de los delitos de nuestro tiempo los cometen profesionales que fueron formados en las aulas universitarias. Al igual que Nagao, nos preguntamos ¿cómo podría la universidad fortalecer la formación ética y humana en una sociedad donde prevalece sobre cualquier cosa el valor del dinero? ¿Será necesario superar la universidad profesionalizante heredada de las corrientes pragmáticas de la educación superior?

Luc Weber, de la Universidad de Ginebra, en su ponencia titulada Financial Managemente and Planning or How to Implement Changes More Smoothly, coincidió con la tesis general de que las universidades tienen el reto de enfrentar cambios de magnitudes inéditas en su historia. Señaló que en este tiempo se tienen que asumir transformaciones radicales en la naturaleza de sus estudiantes, en la forma como se entrega el conocimiento y se hace investigación, y en el modo como se interactúa con la sociedad civil, con el mundo de los negocios, con el Estado y con otras universidades. Y que, por si esto no fuera suficiente, también se están presentando cambios dramáticos en la forma como se administran las finanzas y los recursos humanos en las universidades.

Para Weber, pese a que se están haciendo esfuerzos significativos para cambiar la estructura organizacional y el gobierno de las universidades tradicionales, esto parece no ser suficiente para llevar a cabo las tareas urgentes de la educación superior de nuestro tiempo debido a que el impacto de la globalización y de las tecnologías de la información y la comunicación ha sido tan rápido y profundo que las universidades aún no logran encontrar el rumbo a seguir para hacer posible la educación superior del futuro. Subrayó que mientras las empresas y los gobiernos hoy hacen grandes esfuerzos por adaptarse a los difíciles y rápidos cambios provocados por la globalización y las TIC, las universidades cambian lentamente y que para acelerar el cambio:

- $\quad$ Es preciso que las universidades sean más sensibles a las necesidades de sus estudiantes y países.

- Se debe romper el monopolio de la enseñanza favoreciendo el surgimiento de múltiples instituciones públicas y privadas que ofrecen educación superior.

- La universidad debe ratificar su responsabilidad ante la humanidad, ya que es la única institución que a la fecha es capaz de preservar y transmitir el patrimonio cultural de la sociedad, pues cuenta con las competencias profesionales y el status apropiado para analizar los problemas sociales en forma independiente, crítica, responsable y científica.

Igualmente, considera Weber que es preciso garantizar la efectividad de los gobiernos universitarios, ya que la vieja 
estructura de excesiva consulta en múltiples instancias y órganos de la institución vuelve lentos los procesos de toma de decisiones y es proclive a la obstaculización en cada instancia donde los asuntos son tratados. La vieja estructura en la toma de decisiones, tiene que ser reemplazada por la descentralización y la ejecución autónomas al interior de la universidad.

Los cambios profundos en la estructura y organización de las universidades tienen como correlato la redistribución del poder $\mathrm{y}$, en consecuencia, ante la reforma impera el conservadurismo, por lo que suele haber serias resistencias al cambio y la innovación. Si bien el sistema de gobierno consultivo fue funcional en el pasado, actualmente muestra serias deficiencias, por lo que se requiere un incremento de la autoridad en los mandos medios y, políticas y estrategias de descentralización. Para Weber, la Planificación Estratégica constituye un sistema de gestión que combina la participación y la autoridad para la efectiva ejecución, que sería muy útil en educación superior.

$\mathrm{Al}$ respecto, es importante señalar que en el 2005 las autoridades de la Universidad de Costa Rica, en la resolución de la Vicerrectoría de Docencia VD-R7706-2005, acordaron la elaboración de un Plan de Desarrollo orientado a los Recursos Humanos Académicos desde el enfoque de la Planificación Estratégica que está siendo implementado en la institución en forma obligatoria, bajo la asesoría del Centro de Evaluación Académica y en coordinación con la Oficina de Planificación Universitaria.

\subsection{Algunos ejemplos de cambio y gestión del cambio en los países miembros del Proyecto Seis Naciones}

Kenneth Mortiner, de la Universidad de Hawai, en su ponencia titulada The Strategic Planning Process at the University of Hawaii, describe la experiencia del proceso de Planificación Estratégica que se llevó a cabo en su universidad. Considera que fue exitosa en términos generales y que los resultados más importantes fueron la toma de conciencia de la necesidad del desarrollo y mejoramiento de la profesionalización del personal docente, y la cooperación interuniversitaria, ya que la Universidad de Hawai tiene sedes en varias islas. Además, se dio especial importancia a la necesidad de fortalecer e incrementar la cooperación internacional.

Linda Low, de la Universidad Nacional de Singapur, en su ponencia Singapore's Experience in Higher Education, hizo una reseña de las instituciones de educación superior en su país, que se constituyó como tal recientemente, en 1965, cuando se separó de Malasia para convertirse en una nación soberana. Señaló que desde sus orígenes las universidades en su país estuvieron orientadas al desarrollo de la ciencia y la tecnología y que debido al interés por articular la formación universitaria al desarrollo científico y tecnológico, periódicamente se hacen estudios de seguimiento de graduados y con empleadores que han mostrado ser un excelente mecanismo para que las universidades cumplan con tal objetivo.

Otro aspecto fundamental de las universidades en Singapur es el propósito explícito de ser instituciones de primera clase, razón por la cual se desarrollan planes de estudio que enfatizan la creatividad, la invención y el desarrollo de tecnologías. También, existe una política de aprovechamiento de talentos, para favorecer la permanencia en la universidad y en el país de estudiantes de alto rendimiento, ya que son conscientes de que compiten con universidades occidentales de mucho prestigio, particularmente con universidades norteamericanas, que tienen políticas agresivas de aprovechamiento de talentos extranjeros.

En cuanto a la tecnología, se han hecho importantes esfuerzos por explotar las oportunidades que dan las tecnologías 
de la información y la comunicación en la enseñanza bimodal y a distancia, y se han desarrollado sólidos programas de educación continua y reciclaje profesional. Estos esfuerzos se enmarcan dentro de la política de Singapur de posicionarse en los niveles nacional e internacional como un país orientado a la educación.

Naoky Murata, Ministro de Educación de Japón, en su ponencia titulada Reform Measures for Universities in the 21th. Century, ofreció un panorama general de la educación en Japón y subrayó que después de la Segunda Guerra Mundial, las universidades lideraron la industrialización de la economía y el desarrollo de la fuerza de trabajo calificada que permitió que Japón se colocara entre los países más desarrollados y ricos del mundo. Treinta años después, debido a que la sociedad del nuevo siglo requiere grandes cambios y a que el mismo Japón está cambiando, incluso demográficamente debido a la caída significativa en la tasa de natalidad, hay conciencia de que también está cambiando la estructura de la fuerza laboral y de la industria y que, por ende, la educación superior debe cambiar.

Señala Murata que para responder a las grandes reformas y tareas que debe asumir la educación superior de Japón para el siglo XXI, se han implementado programas para mejorar la docencia y elevar la calidad de la investigación. También, se le ha brindado una gran importancia a la educación continua, debido a que:

- Cada vez más personas requerirán de nuevas habilidades y destrezas, así como de procesos de reciclaje profesional.

- Por las características del estudiantado no tradicional se debe permitir una mayor flexibilidad horaria y curricular.

- Es necesario diseñar programas abiertos con cursos específicos que obtengan créditos que se puedan acumular para optar por algún grado académico o especialidad.
Para Murata, las universidades del futuro se caracterizarán por una mayor interdisciplinariedad y por el trabajo comprehensivo, porque serán capaces de acercarse más a la sociedad para atender necesidades, problemas y demandas concretas, y porque desarrollarán sistemas robustos y oportunos de rendición de cuentas.

\section{Las tecnologías de la información y la comunicación (TIC) y su impacto en la sociedad y en la universidad del siglo XXI}

Debido a que las nuevas tecnologías y su impacto en la sociedad y en la educación superior fueron un tema específico de la conferencia (Tema: El papel de las universidades en el crecimiento económico; Educación superior y tecnología: ¿cuándo cambiará realmente la tecnología, cómo se enseña y cómo aprenden los estudiantes en la universidad?), presentamos a continuación las principales ideas de las ponencias que abordaron este tópico.

Stephen Holding, Decano de Margan Stanley Investment Management, en su ponencia Promoting Financial Efficiency through Administrative Technology Aplications, señaló que en su institución, con el propósito de hacer una adecuada reforma académica, se consideran fundamentales los siguientes aspectos:

- $\quad$ El cambio en la cultura administrativa.

- Mantener la educación superior accesible.

- La innovación tecnológica.

- Contar con una fuerza de trabajo flexible, en función de las necesidades institucionales.

- Tener como matriz de gestión un sistema flexible, colaborativo y empresario. 
Para Holding las TIC son una oportunidad para elevar la eficiencia administrativa y mejorar la calidad de los servicios que brinda la institución. Además, se adhiere a la tesis de que las universidades deben ser "empresarialmente inteligentes" y aprovechar las oportunidades que se abren para las alianzas estratégicas con el sector público y privado. A su parecer, la venta de servicios es una excelente oportunidad para sanear las finanzas de las instituciones de educación superior, con el valor agregado de que vinculan la universidad con las empresas y la sociedad.

Robin H. Beck, de la Universidad de Pennsylvania, en su ponencia $E$ Nabled Information at the University of Pennsylvania, plantea que las TIC han sido un disparador de las nuevas fuerzas que gobiernan el mundo contemporáneo. Por esta razón, sostiene la tesis de que estas tecnologías son un elemento que puede ser muy útil para dar solución a algunos de los nuevos problemas que enfrentan las universidades en nuestro tiempo. Afirma que los empleados de hoy tienen el reto de ser cada vez más inteligentes y eficientes y, por consiguiente, las personas que desean encontrar buenos trabajos deben, a su vez, ser más inteligentes y eficientes. Una forma de agregar inteligencia y eficiencia es la formación universitaria y postuniversitaria y para ello se deben explotar al máximo las ventajas y características de las TIC.

En la misma línea de pensamiento de Beck, Darren Rushworth, representante de Oracle Global Learning Initiatives, en su ponencia Internet Changes Everything, se refirió específicamente a la Internet. Aclaró que para que un medio de comunicación sea considerado un medio masivo, debe tener al menos una audiencia o un número de subscriptores de 50 millones de personas y que, por ejemplo, a la electricidad le tomó 46 años alcanzar ese nivel de subscriptores, a la radio 22 , a las computadoras 16 , y a la Internet sólo 4 años. La Red de Redes es el primer medio de comunicación masiva que históricamente alcanza este nivel en menos de cinco años. En la actualidad, con un crecimiento sin precedentes, alcanzó 100 millones de subscriptores en cinco años, y su tráfico se duplica cada 100 días. Además, también está superando al teléfono, ya que en 1999 se envió más información por este medio que por vía de la voz por primera vez en la historia de las telecomunicaciones. El siguiente cuadro ilustra el comportamiento de estos y otros medios de comunicación, y el tiempo que tardaron en convertirse en medios masivos.

Cuadro No. 1

Período de masificación de algunos medios de comunicación

\begin{tabular}{clc}
\hline Fecha & Invención & $\begin{array}{c}\text { No. de años } \\
\text { para convertirse } \\
\text { en un medio masivo }\end{array}$ \\
\hline 1873 & Electricidad & 46 \\
1876 & Teléfono & 35 \\
1886 & Automóvil & 55 \\
1906 & Radio & 22 \\
1926 & Televisión & 26 \\
1975 & Computadora Personal & 16 \\
1983 & Teléfono móvil & 13 \\
1994 & La Web & 04 \\
\hline
\end{tabular}

Fuente: Newsweek, 1998.

Para Rushworth la aceptación de la Internet en educación superior crece también rápidamente y ello implica un reto a la administración universitaria y a la forma como se enseña y se aprende en este nivel. Entre los retos administrativos señala el diseño de procedimientos eficientes e innovadores para dar respuestas pertinentes y adecuadas a las siguientes tendencias:

a. Cambios demográficos: estudiantes no tradicionales (demanda por educación continua y por reciclaje profesional). Envejecimiento de la población. Aumento de la población estudiantil, mientras los presupuestos estatales se reducen. 
b. Incremento de la competencia nacional e internacional: cada vez más instituciones nacionales $\mathrm{y}$ extranjeras crecen y ofrecen opciones a los eventuales estudiantes, tanto nacionales como extranjeros.

c. Aumento de las demandas de los estudiantes: los estudiantes deciden dónde estudiar, esto les da un gran poder.

d. Nuevos proveedores de educación: las universidades de las empresas.

En este entorno de cambios Rushworth propone que las TIC pueden ayudar a resolver los problemas que estas tendencias introducen, ya que, por ejemplo, en el nivel administrativo pueden proveer sistemas de información eficientes, de bajo costo final, que orienten en forma sostenible y segura la toma de decisiones en forma oportuna y adecuada. También, hacen posible elevar la calidad y productividad administrativas, contribuyendo así a mejorar los servicios a los clientes internos y externos de la universidad porque permiten $\boldsymbol{e l}$ autoservicio en el nivel administrativo; es decir, la consulta personalizada de información académico-administrativa, del expediente académico, de la matrícula y de notas, entre otros. Además, dan acceso rápido y eficaz a información a quienes la necesitan, ya sean administradores, docentes, investigadores o estudiantes.

Para Rushworth las TIC también son una oportunidad para enfrentar el reto de transformar los procesos de enseñanza y aprendizaje, así como el diseño y planificación de los planes de estudio. Señala que el acelerado cambio en el plano tecnológico hace obsoletos rápidamente los currículos y que la vida útil de las carreras hoy es realmente efímera. Por ejemplo, en áreas técnicas la vida promedio de un grado académico es de sólo tres años, situación que hace indispensable que la universidad atienda las necesidades de reciclaje profesional y que reduzca al máximo los currículos, a fin de hacer la obtención del grado lo más rápida posible, dejando abierta la posibilidad del reciclaje. Además, debido a que para participar y tener éxito en la Sociedad de la Información las personas deben saber cómo usar las computadoras para comunicarse y tener acceso al mundo económico actual, las universidades deben incluir en sus procesos de formación la alfabetización en el uso de estas tecnologías para todos los estudiantes y no sólo para los de áreas o carreras tecnológicas.

Rushworth sostiene que la clave de la educación del futuro es la calidad y que es un deber de la educación de nuestro tiempo proveer calidad educativa a todas las personas, en cualquier lugar y en cualquier momento. Esto es más importante aún si se toma en cuenta la creciente demanda del sector empresarial por mejorar la educación superior, ya que en la actualidad muchos estudiantes egresan sin tener las habilidades, conocimientos y destrezas que requieren los nuevos puestos de trabajo que crea una brecha cada vez mayor entre las características de los graduados y los trabajadores que demandan las empresas y la industria. Se requiere, por lo tanto, un vínculo más estrecho entre los sectores productivos y la universidad.

Rushworth plantea que la educación continua es una nueva área académica y profesional que todavía no está suficientemente desarrollada en las universidades y que es urgente atender, ya que se debe dar respuesta a la tendencia del mercado laboral de nuestro tiempo hacia una alta competitividad laboral, por lo que las empresas valoran el desarrollo académico de sus trabajadores. Esta tendencia hace que cada vez más personas ya graduadas se interesen por continuar sus procesos de especialización y formación universitaria. En la implementación de sistemas robustos y apropiados de educación continua, Rushworth propone que las TIC, también en este caso, son una respuesta viable para atender las nuevas necesidades educativas de la población adulta, sin que tenga que 
enfrentar las limitaciones que imponen las barreras del espacio y del tiempo. La educación en línea y la bimodal son opciones promisorias para las universidades del siglo XXI.

En relación con el trabajo de aula y los sistemas presenciales, Rushworth opina que los sistemas multimedia elevan la calidad de la enseñanza, ya que permiten que más sentidos intervengan en el proceso de aprendizaje, por lo que las posibilidades de motivación e involucramiento del estudiante son mayores. Además, hizo un llamado de atención sobre el hecho de que la pizarra y la tiza anteceden la invención de la imprenta -que se considera el mayor cambio en los medios de enseñanza y aprendizaje ocurrido antes de la invención de las TIC (Briggs \& Burke, 2002; Burke \& Ornstein, 1997; McLuhan, 1994, 2000; Olson, 1999-, y afirma que hoy no hay excusas para no utilizar los nuevos recursos disponibles para la enseñanza y el aprendizaje.

Charng-Ning Chen de la Universidad Tecnológica de Nonyang de Singapur, en su ponencia Technology and the Curriculo: The NTU Expirience y Yoshiyuki Naito, del Instituto de Tecnología de Tokio, con su ponencia Science and Technology in Universities of the 21th. Century, hicieron énfasis en el interés de Singapur y Japón por desarrollar en las universidades de sus países la ciencia y la tecnología, ya que reconocen que estos aspectos juegan un papel fundamental en el desarrollo económico de sus naciones. En particular, nos parece interesante la propuesta de Naito, quien plantea que Japón se ha destacado en las últimas décadas por sus aportes a la ciencia y la tecnología, teniendo como competidores sólo a los Estados Unidos y a Alemania en el desarrollo de dispositivos de consumo masivo como la cámara fotográfica, la radio, la televisión, las videograbadoras, los hornos eléctricos y los automóviles. Sin embargo, esta tendencia ha ido decreciendo debido a que en Japón se presenta en los últimos años una reducción significativa de estudiantes interesados en seguir carreras científicas y tecnológicas. Por esta razón, se están haciendo importantes esfuerzos por desarrollar planes de estudio en las universidades japonesas que estimulen el interés por las áreas científicotecnológicas y, particularmente, que eleven la creatividad.

Para Naito, la creatividad es un concepto complejo, razón por la cual propuso una definición propia para aclarar a qué se refería cuando hablaba de estimular la creatividad en los estudiantes de educación superior:

a. La creatividad implica proponer una nueva estructura, una nueva idea o una nueva manera de pensar. Esto implica que en la creatividad está presente algo "nuevo".

b. La creatividad supone la concreción de la idea innovadora, es decir, la creación de prototipos y de los mecanismos necesarios para su respectiva prueba.

c. Además, la creatividad supone la implementación; es decir, la clarificación de los potenciales usos que tendría el nuevo dispositivo.

d. Luego de estas etapas, es necesario hacer una propuesta de producción del dispositivo para ponerlo al servicio de los consumidores.

e. Finalmente, la creatividad debería permitir nuevas propuestas para mejorar lo hecho e, incluso, superarlo.

Como ejemplo de este proceso, Naito se refirió al descubrimiento de las ondas electromagnéticas que hizo posible el desarrollo de las radiocomunicaciones y las antenas, luego de las antenas lineales y de superficie $\mathrm{y}$, finalmente, el perfeccionamiento de la antena lineal que hizo posible el desarrollo de la antena parabólica. Como es evidente, este proceso involucra tanto a científicos como a ingenieros, ya que implica el desarrollo de conocimiento puro y aplicado.

Para Naito, volver a colocar a Japón a la vanguardia de la producción científica 
y tecnológica es una tarea que empieza mucho antes de que los estudiantes ingresen a la universidad. Primero, es necesario mejorar la educación primaria y secundaria para elevar el potencial interés de los estudiantes por seguir carreras de ciencia y tecnología. Luego, se debe incrementar el número de estudiantes de las áreas científicas y tecnológicas en las universidades. Finalmente, es necesario implementar planes de estudio y procesos de enseñanza y aprendizaje en las universidades que permitan elevar los niveles de creatividad en el estudiantado.

\section{Conclusiones en los cuatro temas tratados en Conferencia}

A continuación, se presentan las principales ideas y conclusiones de los cuatro temas que se trataron en la Conferencia. Cada tema corresponde a un subtítulo con el propósito de dar una perspectiva global de cada uno de ellos.

\subsection{Políticas en Educación Superior}

En esta sesión se discutió la situación de la institución universitaria en el marco de las macrotendencias económicas, sociales, políticas, y tecnológicas que afectan a la educación superior, ya que dichas macrotendencias se deben considerar para implementar políticas universitarias adecuadas en el futuro cercano, que den respuesta a las demandas actuales de educación superior de calidad, pertinente, equitativa y oportuna. $\mathrm{Al}$ respecto, una de las ideas compartida por los participantes fue que la institución universitaria está en un proceso de transición, consecuencia del paso de la sociedad industrial a la postindustrial, en el que se están haciendo los ajustes necesarios de la misión, visión y acciones de la universidad para responder a la nueva sociedad que no está en el futuro, sino que ya es una realidad.
Con base en lo anterior, se llegó a la conclusión de que en este momento de cambio aún no es del todo claro cuál debe ser la misión de la universidad en el escenario de la Sociedad del Conocimiento, ya que hay confusión entre la misión histórica de la institución y las nuevas tendencias y presiones del mercado y la sociedad sobre el quehacer académico. Es decir, aún no hay consenso sobre si las universidades deben seguir orientando su quehacer con base en su misión tradicional, o deben empezar procesos de transformación administrativa y académica que las sitúen en condición de ser "mercantilmente inteligentes". En relación a este aspecto, se hizo referencia a la clara orientación utilitarista y pragmática de las universidades en China y Singapur.

Por otra parte, está el problema del financiamiento de la educación superior, en una época donde la reducción del aporte estatal es más que importante y probablemente siga disminuyendo, se incrementa la presión por el desarrollo de mecanismos para la generación de recursos propios, con el propósito de permitir que la universidad cuente con los ingresos necesarios para continuar operando de manera apropiada. Quizá, la situación más difícil en relación con el financiamiento sea que en el corto plazo no va a haber más alternativa que elevar sustancialmente el pago de matrícula y los costos asociados a la educación superior que paga el estudiante. Esto, sin lugar a dudas, involucra una revisión profunda de la misión, visión y objetivos de la educación superior pública del pasado reciente.

Otro factor a considerar en la dimensión política es el crecimiento progresivo de instituciones de educación superior privadas, por lo que la gran diversidad de instituciones universitarias exige mecanismos permanentes, eficientes y eficaces para el control, la auditoría y la evaluación de las universidades. Si la calidad es un requisito de la educación del futuro, refrendar esa calidad es una obligación políticamente ineludible. Además de la creciente presencia de instituciones de educación superior privada, se 
debe enfrentar políticamente el fenómeno de la internacionalización de la educación superior, ya que es muy probable que en el futuro cercano se incremente aún más el tránsito de estudiantes de grado, postgrado y especialidades entre universidades y puestos de trabajo de distintos países, por lo que es necesario crear estrategias para dar respuesta a la certificación de títulos y estudios universitarios entre las naciones.

En el escenario de la competencia nacional e internacional entre universidades públicas y privadas por recursos financieros y estudiantes es imprescindible revisar la misión, visión y acciones de esta institución, con el propósito de eliminar las tensiones internas y externas que hoy la sumen en una crisis política e ideológica que en algunos momentos parece no tener salida. Al respecto, señaló Noel F. McGinn, de la Universidad de Havard, que sólo cincuenta organizaciones que funcionaron en Occidente hace 500 años, lo hacen hoy: la Iglesia Católica, el Parlamento Inglés y las 48 instituciones restantes son universidades. Esto pone en evidencia que la universidad es una institución que debe su supervivencia a su capacidad de respuesta y adaptación a las nuevas demandas sociales. Para McGinn, hoy, al igual que hace 500 años, el conocimiento resulta un recurso indispensable para el desarrollo y curso normal de la vida y la sociedad, por esta razón considera que las universidades no van a desaparecer, sino que, con base en su misión histórica, lograrán ser mercantilmente inteligentes y políticamente sabias.

Para McGinn, lo más importante es aprender de la propia experiencia $\mathrm{y}$, al igual que los demás participantes, confía en que la universidad, en tanto institución social necesaria y con un amplio patrimonio histórico, es capaz de hacer los ajustes necesarios para, sin olvidar su misión, seguir contribuyendo a resolver los problemas de la sociedad y al desarrollo de la cultura en nuestro tiempo.

Shigetaka Imai, de la Universidad de Hiroshima, destacó que para que la universidad continúe cumpliendo con su misión histórica en el corto plazo se deben alcanzar las siguientes metas:

a. Encontrar alternativas de solución a las crisis resultado del conflicto entre universidades y gobierno, universidades públicas y privadas, universidades y mercado.

b. Alcanzar y mantener la eficiencia en la gestión de las universidades y desarrollar planes para sistemas de educación continua.

c. Rescatar la ética en la actividad científica y tecnológica.

d. Considerar que el éxito en el mercado, no necesariamente implica logros éticos.

e. Encontrar alternativas de solución al conflicto actual entre equidad $y$ financiamiento, $y$ entre intereses sociales e intereses de mercado. Se debe reflexionar qué se entiende por equidad en nuestro tiempo.

f. No perder el norte ético, la autonomía y la criticidad que han caracterizado a la universidad como institución libre y defensora del desarrollo del pensamiento social y culturalmente crítico y responsable.

g. Reflexionar si en la actualidad, con las condiciones que prevalecen de contratación de los docentes por tiempo definido o a plazo se puede promover el pensamiento libre, creativo y crítico. (Report of the Six-Nation Higher Education Project, 2000, p. 159).

\subsection{Gestión estratégica para las universidades}

Debido a los problemas de gestión de la universidad en nuestro tiempo y al papel crítico que juega la administración universitaria en el marco de los cambios y tareas urgentes para ajustar el quehacer de la educación superior a las demandas de la sociedad actual, se concluyó que la Administración Estratégica es una opción 
viable para las universidades, ya que se ajusta a las características consultivas y participativas de la estructura tradicional del gobierno universitario, pero agiliza la toma de decisiones e incorpora la evaluación y el control como partes esenciales del proceso de gestión, lo que permite el aseguramiento de la calidad, la rendición de cuentas, la eficiencia y la eficacia, tanto en el nivel administrativo como en el académico.

\subsection{Educación superior y sociedad}

Entre los temas más relevantes de la relación entre educación superior y sociedad, además de la globalización económica y del impacto de las TIC, se analizó la internacionalización del mundo de los negocios y la influencia del mercado en las universidades, ya que estos factores producen tensión entre la universidad y la academia y entre la universidad, la sociedad y el mercado. Es un hecho que la internacionalización de los negocios y la influencia del mercado van a mantenerse y, probablemente, a incrementarse en el futuro. Por esta razón, se deben crear mecanismos tales que, sin ir en detrimento de la misión histórica de la universidad, permitan aprovechar los vínculos con los sectores productivos para contribuir al desarrollo económico y para obtener recursos propios.

Quienes administran las universidades contemporáneas deben ser inteligentes y aprovechar la demanda creciente por educación superior y por investigación de alto nivel. La educación continua y la apertura para hacer alianzas estratégicas con los sectores productivos son formas de responder a las nuevas demandas socioeconómicas y medios legítimos para resolver la apremiante situación financiera que enfrentan las instituciones de educción superior en la actualidad.

Se concluyó que la calidad es la clave del éxito para las universidades del futuro. Las personas, las empresas y los gobiernos necesitan la garantía de que pueden acceder a universidades de alto nivel, cuyo prestigio se funda en los resultados de sus egresados en el mundo del trabajo y, en la relevancia de la investigación y la acción social que realizan.

\subsection{El papel de las universidades en el crecimiento económico; Educación superior y tecnología: ¿cuándo cambiará realmente la tecnología?, ¿cómo se enseña y cómo aprenden los estudiantes en la universidad?}

Todos los participantes de los países del Six-Nation Program estuvieron de acuerdo en que es urgente la incorporación de las TIC en educación superior; por esta razón, la formación del profesorado en el uso de estas tecnologías para mejorar la docencia, la investigación y la acción social es una tarea prioritaria. Fue una idea de consenso que, debido a sus características, estas tecnologías no sólo son una herramienta para hacer la oferta académica más flexible, sino también, si se adaptan adecuadamente, pueden contribuir a un cambio significativo en la enseñanza y el aprendizaje en la universidad.

Las TIC hacen posible el desarrollo de sistemas en línea, a distancia y bimodales que resultan más convenientes a los estudiantes no tradicionales. También, como se expuso anteriormente, el uso de estos recursos en la enseñanza presencial puede elevar substancialmente la calidad de los aprendizajes, el interés y la motivación de los estudiantes. Además, las TIC se pueden utilizar en el nivel administrativo para mejorar la calidad de los servicios y hacer la gestión más eficiente y eficaz. Intra-redes, páginas Web, correo electrónico, teleconferencias y otros servicios en red, tanto internos como externos a la institución, podrían facilitar el ideal de "oficinas sin papeles", "autoservicio" y acceso rápido y cómodo a la información y a las personas, lo que contribuiría al ahorro de papel, mayor eficiencia, calidad de servicio y eficacia. 
Sin duda alguna las TIC son el signo distintivo de nuestro tiempo, por esta razón están presentes en todas las actividades sociales y productivas en la actualidad. Obviar este hecho, particularmente en lo que concierne al mundo del trabajo y a la economía global es insostenible, en especial en una institución que tiene como propósito principal preparar la fuerza de trabajo profesional y especializada.

\section{A manera de conclusión}

El abordaje que se hizo de los principales problemas de las universidades de los seis países miembros del proyecto, no sólo fue profundo, serio y autocrítico, sino que se ofrecieron alternativas de solución para algunas de las principales dificultades y dilemas de la educación superior en nuestro tiempo. Pese a las grandes diferencias socioeconómicas, políticas y culturales, estos países enfrentan, como se pudo ver, problemas similares que, a su vez, tienen soluciones semejantes, cuyas claves son: $l a$ innovación y flexibilidad administrativa y académica, el aprovechamiento de las tecnologías de la información y la comunicación, la orientación en la gestión al cliente interno y externo, la cooperación local e internacional y el contacto con la sociedad.

A nuestro parecer, entre las lecciones más importantes que deja este esfuerzo de cooperación universitaria de seis de los países más ricos y desarrollados del mundo está el reconocimiento de que las instituciones de educación superior aisladas, ajenas al contexto nacional e internacional y aferradas a la tradición parecen estar destinadas al fracaso.

\section{Notas}

1. En inglés:

- Framework for Higher Education Policy.

- Strategic Management for Universities.

- Higher Education and Society.
- The rol of the Universities in Economic Growth; Higher Education and Technology: When Will Technology Really Change How Universities Teach and Students Learn). (Report of the Six Nation Higher Education Projet, 2000, p. 2).

2. Experto de la Universidad de Kassel, Alemania, quien participó en la conferencia con la ponencia Reform for Higher Education in the 21th. Century. La misma fue incluida en el documento final de la conferencia, titulado Report of the Six-Nation Higher Education Project (March, 2000).

\section{Referencias bibliográficas}

Briggs, Assa y Burke, Peter. De Gutenberg a Internet. Una historia social de los medios de comunicación. España: Taurus, 2002.

Burke, James y Ornstein, Robert. The Axemaker's Gift. Technology's Capture and Control of Our Minds and Culture. U.S.A.: Tarcher Penguin, 1997.

Jofré, Arturo. La Universidad en América Latina. Desafios y estrategias para las próximas décadas. Cartago: Editorial Tecnológica de Costa Rica, 1994.

McLuhan, Marshall. Understanding Media. The Extensions of Man. U.S.A.: MIT Press, 1994.

McLuhan, Marshall. The Gutenberg Galaxy. The making of typographic man. Canada: University of Toronto Press, 2000.

Olson, David. El mundo sobre el papel. El impacto de la escritura y la lectura en la estructura del conocimiento. España: Gedisa, 1999.

Research Institute for Higher Education of Hiroshima University. Higher 
Education Reform for Quality Higher Education Management in the 21th. Century. Economical, Technological, social and political Forces Affecting Higher Education. Japan: Takatoo Print Media, 2000.
Tünnermann, Carlos. La educación superior frente al cambio. San José, Costa Rica: EDUCA-CSUCA, 1997.

Tünnermann, Carlos. Educación superior de cara al siglo XXI. San José, Costa Rica: Mirambell, 1999. 